\title{
Controlling intershell rotations in mesoscopic electron clusters
}

\author{
V. Golubnychiy \\ Universtität Rostock, Fachbereich, Universtitätsplatz 3, D-18051 Rostock, \\ Germany \\ P. Ludwig, A.V. Filinov, and M. Bonitz* \\ Christian-Albrechts-Universität zu Kiel, Institut für Theoretische Physik und \\ Astrophysik, Leibnizstrasse 15, 24098 Kiel, Germany
}

\begin{abstract}
A previously proposed idea [1] of controlling the angular mobility of electrons in a quantum dot by addition/removal of a single electron is verified and confirmed by computer simulation. Conditions for realization of the scheme in semiconductors are discussed.
\end{abstract}

Key words: single-electron control, Quantum dots, Wigner crystal

Introduction and idea. Mesoscopic ensembles of a few (10 . . 50) electrons confined in open or closed quantum dots continue to attract increasing interest due to their potential applications as submicron optical or transport devices. A

\footnotetext{
* Corresponding author. Fax: +49.431.880.4094

Email address: bonitz@theo-physik.uni-kiel.de (M. Bonitz).
} 
frequently used model which contains all main features are quasi-2D electrons in a harmonic confinement potential which interact via the Coulomb potential given by the hamiltonian

$$
\hat{H}_{N}=-\sum_{i=1}^{N} \frac{\hbar^{2} \nabla^{2}}{2 m}+\frac{q^{2}}{\varepsilon_{b}} \sum_{i>j}^{N} \frac{1}{\left|\mathbf{r}_{i}-\mathbf{r}_{j}\right|}+\frac{1}{2} m \omega_{0}^{2} \sum_{i}^{N} r_{i}^{2}
$$

In the ground state, the electrons are arranged in concentric shells the configuration and symmetry of which is very sensitive to the precise particle number $N$, e.g. [2]. In ref. [3] we presented a first principle path integral quantum Monte Carlo (PIMC) analysis of the phase diagram of the system (1) and demonstrated that, when the confinement strength $\omega_{0}$ is lowered (at temperature $T>0$ ) a series of structural changes occur, leading from a quantum (Fermi) liquid, over a crystal-like state to a classical Coulomb liquid. The Wigner crystal state is characterized by very strong Coulomb correlations (with the correlation energy exceeding the Fermi and thermal energy by two or more orders of magnitude) and by strong localization of the electrons. It was observed that in the crystal phase spin and quantum diffraction effects (overlap of individual electrons) are weak [1,3]. Electron (or hole) crystallization should be observable in large open quantum dots of high quality at densities of the order of $\left(10^{8} \ldots 10^{10}\right) \mathrm{cm}^{-2}$ and temperatures of the order of $100 \mathrm{mK}$, depending on the material [9].

The mesoscopic "crystals" have a number of unusual properties, including the existence of several crystal phases with either partial (only radial) or complete ordering. The corresponding "melting" transitions occur at characteristic temperatures or densities which are remarkably $N$-dependent. The most striking example is the $9(!)$ orders of magnitude difference of the rotational melting temperatures or densities of the clusters with $N=19$ and $N=20$ electrons 
$[2,3]$. Based on this, in a recent paper we proposed a novel single electron control mechanism where orientational melting is initiated (stopped) by adding (removing) a single electron [1]. This can be accomplished e.g. by injection (removal) through a vertical gate or channel or by tunnel injection (removal ) through the barrier above the dot using the tip of a scanning tunnelling microscope, positioned above the inner shell, see the schematic in Fig. 1. To probe for the existence of relative rotations of the two shells, a weak angular driving force can be applied to the electrons in the dot while pinning the inner shell by an impurity (to prevent the trivial joint rotation of both shells which is possible due to the symmetry of the hamiltonian). This excitation can be generated either by Coulomb drag from a current in a metal ring concentric with the dot in a parallel layer, e.g. [4], or by exciting a persistent current [5] using a normal magnetic field threading the interior of the dot.

We have performed a series of numerical simulations which are intended to verify the idea and to specify the main conditions. Here we present the first results.

Simulation details. The quantum intershell rotation dynamics of the strongly correlated system of $19+1$ electrons, triggered by the injection/removal of one particle, is governed by the von Neumann equation for the $N$-particle density operator $\rho_{N},(N=20)$, e.g. [6]

$$
i \hbar \frac{d}{d t} \rho_{N}-\left[\rho_{N}, \hat{H}_{N}+\Delta \hat{H}_{N}\right]=0
$$

Here $\hat{H}_{N}$ is given by $(1)$ and $\Delta \hat{H}_{N}$ contains the additional contributions due to the external angular excitation, the particle insertion (removal) and dissipation maintaining a constant temperature $T$. Performing a Wigner transform 
of Eq. (2) leads to the equation of motion for the $N$-particle Wigner function [6] which can be identically transformed into an integral equation and an iteration series containing contributions from classical dynamics and all quantum corrections, for details see e.g. $[7,8]$. This scheme allows for a rigorous simultaneous numerical treatment of strong correlation and quantum effects by a combination of molecular dynamics and classical and quantum Monte Carlo methods [7]. The initial condition for the strongly correlated Wigner function is obtained from a path integral Monte Carlo calculation for the 19 electron cluster in its orientationally ordered state as reported in [3], where the temperature is chosen well below the oriental melting point $T_{o}^{19}$. The 20th particle is initially high above the cluster plane, so its initial Wigner function separates from the full function.

As mentioned above, a peculiarity of the orientational (i.e. completely) order mesoscopic crystal is the strong spatial localization. This means, in this state the 19-particle wave function (and hence the Wigner function) consists of 19 well separated sharp peaks [3]. The same applies to the 20-particle cluster after insertion of the electron. Based on this, it is not surprising that the iteration series in terms of corrections to the classical dynamics (see above) rapidly converges, and the dominant contribution arises from the classical trajectories of the localized electrons. This justifies a classical simulation of the intershell rotation dynamics by solving

$$
m_{i} \frac{d^{2} \mathbf{r}_{i}}{d t^{2}}=\mathbf{F}_{i}-\gamma m_{i} \mathbf{v}_{i}+\mathbf{F}_{i}^{e x t}, \quad i=1, \ldots 19
$$

where $F_{i}$ comprises the total force on particle i arising from the potential terms in $\hat{H}_{N}$ (i.e. from the confinement potential and from all other particles). Further, the hamiltonian $\Delta \hat{H}_{N}$, gives rise to the external angular force $\mathbf{F}_{i}^{e x t}$ 
and the dissipative term with damping constant $\gamma$.

Of course, a classical simulation can be expected to capture the basic physics only in situations where neither the external force nor the particle insertion/removal lead to a significant overlap of individual electrons but cause only collective rotations. It turns out that this requirement is easily met in the present case by choosing i) a sufficiently weak excitation and ii) by realization of a slow insertion (removal) of the 20th electron. In our simulations, the first condition was satisfied by using an external force 2-3 orders of magnitude smaller than the force due to the confinement field (this is well below the intershell rotation barrier $U_{o}^{19}$ which we computed beforehand from PIMC simulations) and $\gamma 2-3$ orders smaller than the confinement frequency $\omega_{0}$. The second condition can be achieved by a proper time-dependence of the external field which governs the insertion/removal of the additional electron. Our analysis revealed that during the insertion the wave function of the latter, $\psi_{20}$ under the combined action of the Coulomb repulsion of the 19 particles and the confinement very fast becomes strongly compressed in lateral direction around the classical ground state position $\mathbf{r}_{20}^{0}$. The tunnelling wave packet is spread along the vertical (z-) direction and causes a slow rearrangement of the 19 electrons due to the additional Coulomb potentials $V_{20, i}, i=1 \ldots 19$. The same effect would be caused by an effective charge $e_{20}(t)$ situated at $\mathbf{r}_{20}^{0}$ in the dot plane which increases during the insertion from zero to the elementary charge. The requirement that the Coulomb interactions $V_{20, i}$ are reproduced exactly yields

$$
e_{20}^{i}(t)=\left|\mathbf{r}_{20}-\mathbf{r}_{i}\right|\left[\int_{0}^{z_{20}(0)} \frac{\left|\psi_{20}\right|^{2}\left(\mathbf{r}_{20}, z, t\right)}{\sqrt{\left(\mathbf{r}_{20}-\mathbf{r}_{i}\right)^{2}+z^{2}}}\right]^{-1}
$$


where $z_{20}(0)$ is the initial height above the dot plane and $e_{20}^{i}(t)$ is only weakly dependent on $i$.

Results. A numerical example is shown in Fig. 2 where the effective charge and the time evolution of the tangential current of the outer shell $I_{t}(t)=$ $e \sum_{i=1}^{12} v_{t i}(t) / r_{i}\left(v_{t}\right.$ denotes the tangential velocity component) is plotted. Due to the sub-threshold external force no rotation of the outer shell of the 19particle cluster is possible, only low-amplitude oscillations are observed. At $t=60$ (in units of $\left.T_{0}=2 \pi / \omega_{0}\right)$ the particle injection starts, cf. Fig. 1, the resulting effective charge, Eq. (4), is shown by the dotted line. The simulations indicate that the cluster rapidly adjusts to the ground state configuration $\{1,7,12\}$ of the $N=20$ system. As a result, around $t=75$, i.e. even before the charge reached the dot plane, the outer shell stops to oscillate and instead starts to rotate with respect to the inner shell. The reason is the much lower melting temperature $T_{o}^{20}$ (see above), i.e. the cluster has lost its orientational order and became "mobile" or "rotationally conducting". Next, at $t=110$, the removal of the 20 -th particle is initiated, and it is observed that the rotation quickly stops giving way to angular oscillations again; the cluster has returned to its "off-state". The large oscillation amplitude has its origin in the rotational kinetic energy which is removed from the system by the dissipation mechanism. A much improved signal to noise ratio is observed if the current is averaged over $\Delta t=9 T_{0}$, cf. the full line. In this simulation the initial temperature was chosen about 60 times below the orientational melting point of the 19 particle cluster, $T_{o}^{19}$, which is 7 orders of magnitude above $T_{o}^{20}$. More simulation details are summarized in [11].

Evidently, the figure demonstrates the possibility of a repeated operation of the system. The oscillation amplitude can be drastically reduced by using an 
even weaker external force amplitude, this also reduces the required time for a complete cycle. Let us give estimates for the parameters where this effect will occur in semiconductor quantum dots. The necessary spatial localization allowing for angular ordering of 19 electrons occurs at densities corresponding to $r_{s} \gtrsim 400$, where $r_{s}=\bar{r} / a_{B}$ is the ratio of the average particle separation to the effective Bohr radius $a_{B}=\hbar^{2} \epsilon / m e^{2}$ (for comparison, the partially [only radially] ordered crystal phase appears already for $r_{s} \gtrsim 40$ ) [3]. For electrons in CdTe based dots the effective 2D density is $n \lesssim 2 \cdot 10^{7} \mathrm{~cm}^{-2}$, for GaAs about an order of magnitude lower. A density increase by 1-2 orders of magnitude should be possible in e-h bilayers [9]. For GaAs, the unit of the current is $I_{0} \approx 6 n A$ leading to a rotational current in the range of $(10 \ldots 50) p A$. The time scale is $T_{0}=2.6 \cdot 10^{-11} s$ corresponding to switching cycles in the range of $1 \ldots 10 \mathrm{~ns}$.

Discussion. In summary, strongly correlated states of mesoscopic electron clusters in quantum dots which resemble Wigner crystal behavior lead to interesting collective excitations of the particles. Among them, relative rotation of particle shells is of particular interest for potential applications. This effect turns out to be very robust and extremely sensitive to the precise particle number. Therefore, these rotations can be nontrivially initiated/suppressed by injection/removal of a single electron [1], which has been verified in this paper. Starting from a rigorous theoretical description and computational realization in terms of a Wigner function quantum molecular dynamics $[7,8]$, the strong Coulomb interaction and spatial localization of the electrons allowed us to reduce the simulation to a quasiclassical dynamics. From the analysis of the quantum corrections we expect that the latter as well as a more refined treatment of the particle injection [12] will not quantitatively alter the results. 
Due to the strongly screened Coulomb interaction in GaAs based semiconductor quantum dots, the realization of the proposed single electron control of intershell rotations requires high quality samples and low densities. More favorable conditions exist in materials with strong Coulomb coupling as well as for vertically coupled electron hole dots $[9,10]$.

\section{References}

[1] M. Bonitz, V. Golubnychij, A.V. Filinov, Yu.E. Lozovik, Microelectronic Engineering 63, 141 (2002)

[2] V.M. Bedanov, and F.M. Peeters, Phys. Rev. B 49, 2667 (1994)

[3] A.V. Filinov, M. Bonitz, and Yu.E. Lozovik, Phys. Rev. Lett. 86, 3851 (2001)

[4] See e.g. I.V. Krive, P. Sandström, R.I. Shekher, S.M. Girvin, and M. Jonson, Phys. Rev. B 52, 16451 (1995)

[5] In our case the outer shell electrons exhibit angular coherence. For a discussion of persistent currents in mesoscopic systems, see L. Wendler, and V.M. Fomin, phys. stat. sol. (b) 191, 409 (1995)

[6] M. Bonitz, Quantum Kinetic Theory, Teubner, Stuttgart/Leipzig 1998

[7] See V. Filinov et al., Phys. Rev. B 65, 165124 (2002) and references therein.

[8] Yu.E. Lozovik, A.V. Filinov, and A.S. Arkhipov, Phys. Rev. E 67, 026707 (2003). A similar scheme has been developed in A. Donoso, and C.C. Martens, Phys. Rev. Lett. 87, 223202 (2001)

[9] A. Filinov, M. Bonitz, and Yu.E. Lozovik, J. Phys. A: Math. Gen. 36, 5899 $(2003)$ 
[10] A.V. Filinov, P. Ludwig, V. Golubnychyi, M. Bonitz, and Yu.E. Lozovik, phys. stat. sol. (c) 238, 1518 (2003)

[11] V. Golubnychiy, PhD thesis, Rostock University 2003

[12] P. Ludwig, A. Filinov, and M. Bonitz, to be published 


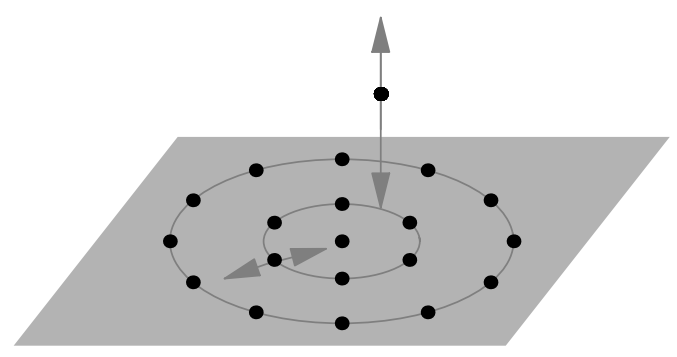

Fig. 1. Scheme of the simulated process. A single electron is slowly inserted into (removed from) the inner shell of the cluster $N=19(N=20)$. To prevent rotation of the cluster as a whole one particle on the inner shell is pinned. 


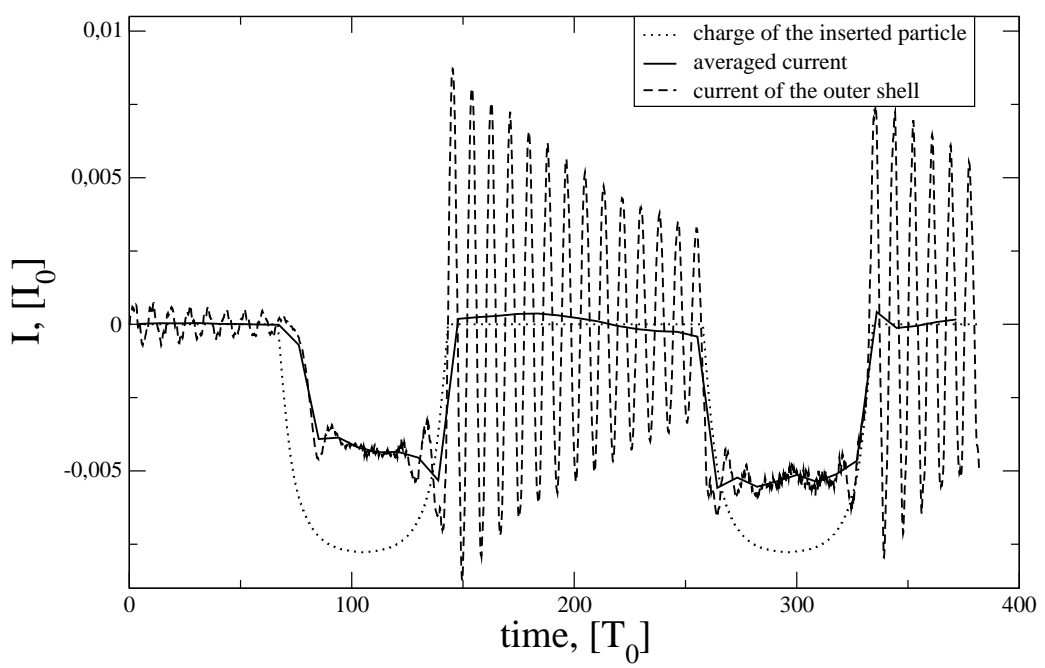

Fig. 2. Angular current of the outer shell (dashed line) and time averaged current (full line) during two electron addition/removal cycles. Dotted line indicates the effective charge $e_{20}(t)$, Eq. (4) of the inserted particle varying between zero and $e_{0}$. Current is in units of $I_{0}=e_{0} / T_{0}$. The damping constant and external force are $\gamma=0.0314 T_{0}^{-1}, F_{0}^{e x t}=2.158 \cdot 10^{-3} F_{o}$, with $F_{o}=m_{e} r_{o} / T_{0}^{2}$ and $T_{0}=2 \pi / \omega_{0}$. 